\title{
Acute gastric mucosal ischemic ulcer: a rare adverse result of peroral endoscopic myotomy
}

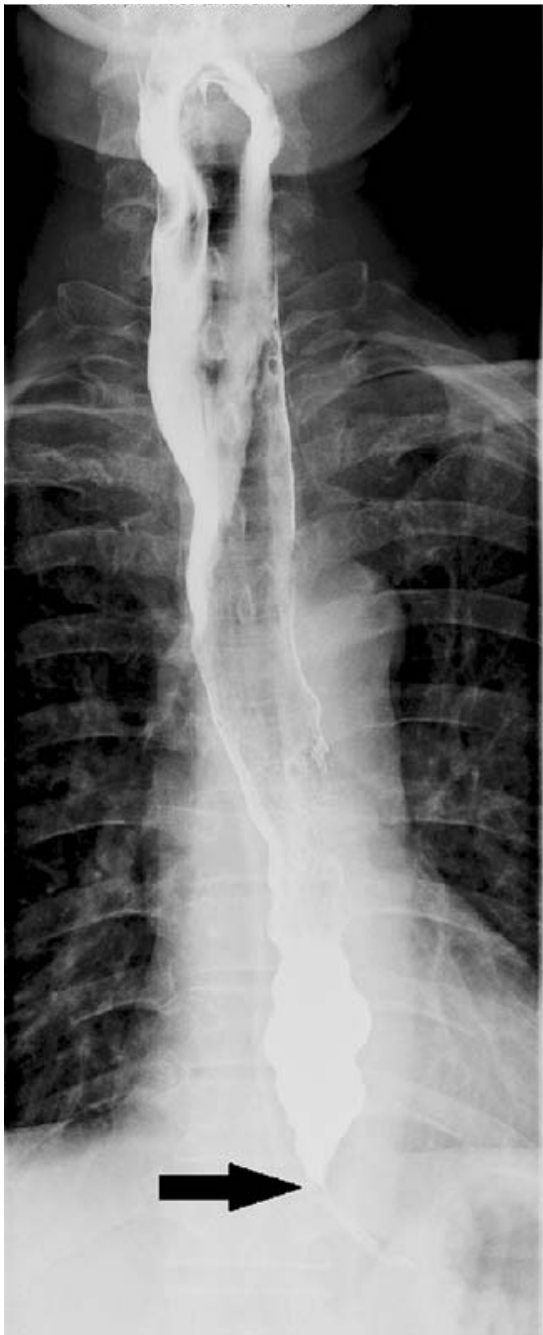

Fig. 1 An esophagogram showing "bird-beak" narrowing of the esophagogastric junction (arrow), consistent with the diagnosis of achalasia.

A 60-year-old man was referred complaining of dysphagia, retrosternal pain, and regurgitation after each meal over the previous 6 years, his symptoms having worsened in the previous year. He was diagnosed with achalasia following examination that included an esophagography ( $\bullet$ Fig. 1). We treated him by performing peroral endoscopic myotomy (POEM) [1]. With the patient under general anesthesia and in the supine position, a submucosal cushion was created on the anterior esophageal wall (2 o'clock position) using a mixture of glycerol solution and $0.01 \%$ epinephrine with indigo carmine, after a vertical mucosal incision had been made

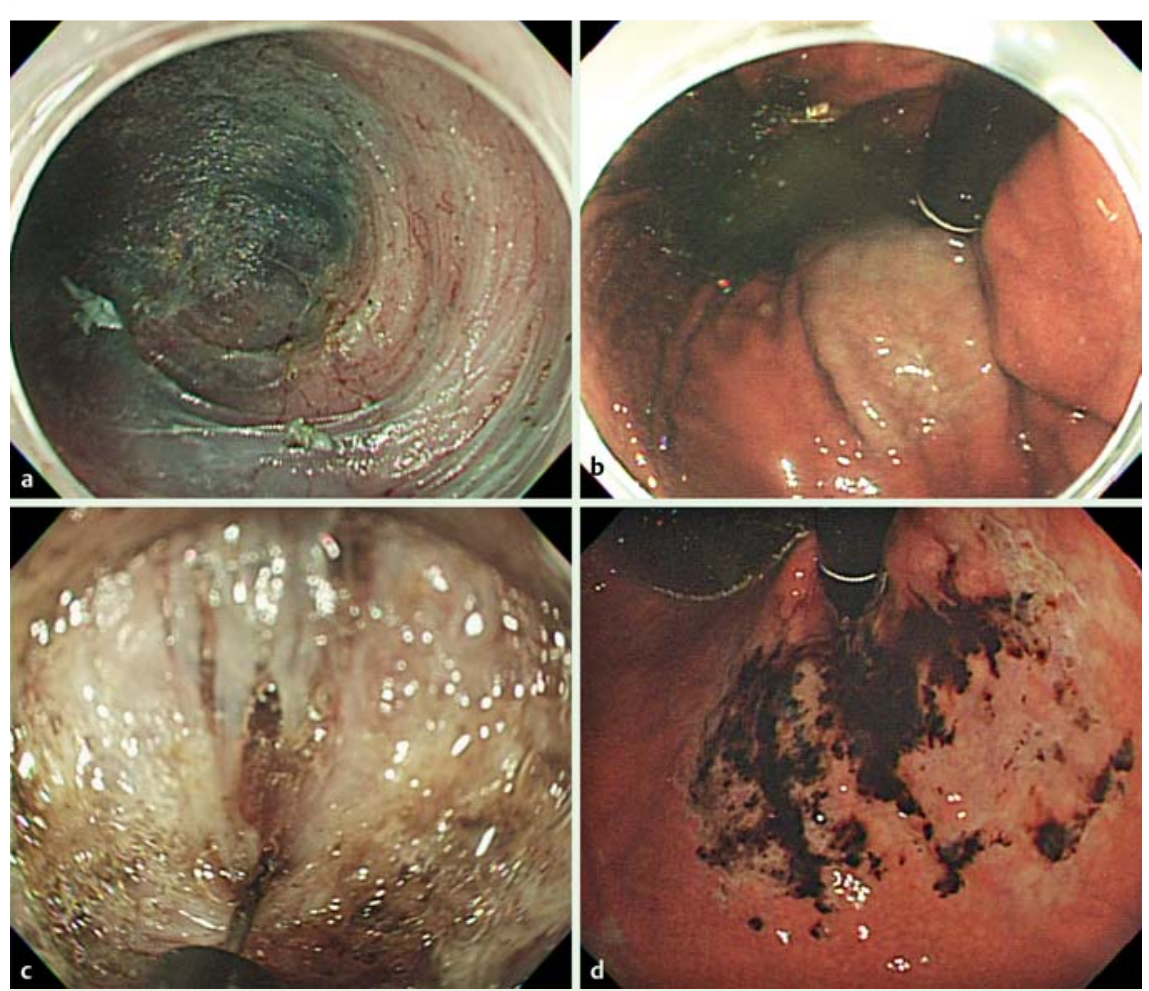

Fig. 2 Endoscopic views showing: a the submucosal tunnel ending in the gastric cardia; $\mathbf{b}$ in reverse view, a blue mucosa over the gastric cardia, indicating where the submucosal tunnel extended beyond the esophagogastric junction; $\mathbf{c}$ selective cutting of the circular muscle bundles from the longitudinal muscle using a triangular tip knife; $\mathbf{d}$ acute ischemic ulcers over the gastric cardia, which were observed after the procedure had been completed.

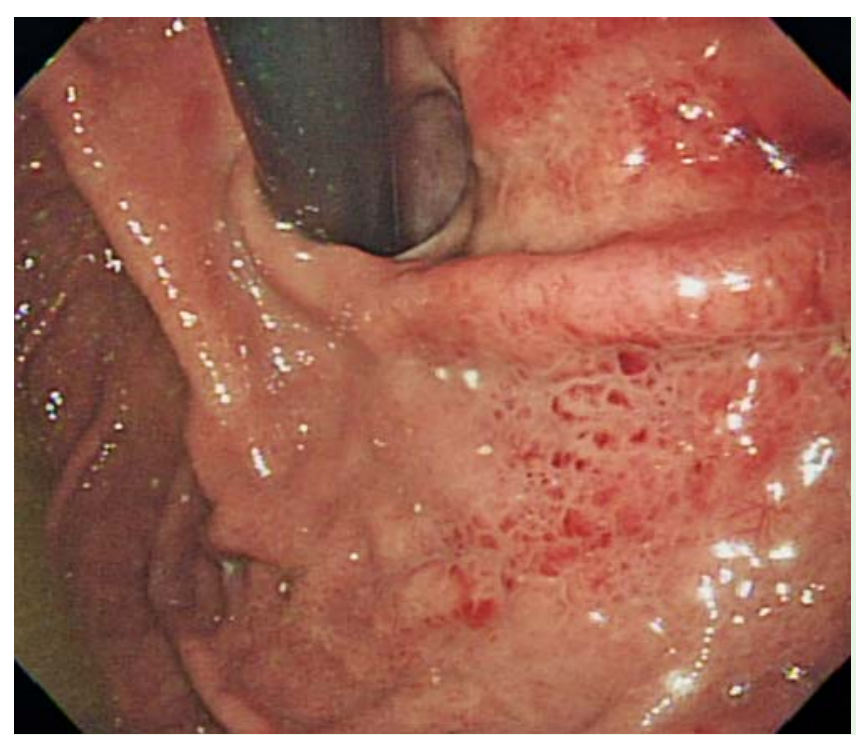

Fig. 3 Image from the endoscopy performed 1 month later showing healing of the previous gastric ischemic ulcers.

with a triangular tip knife (KD640L; Olympus Optical). A gastroscope was subsequently inserted into the submucosal space, and dissection was performed to create a submucosal tunnel that extended
$3 \mathrm{~cm}$ beyond the esophagogastric junction ( Fig.2a, $\bullet$ Fig.2b). We subsequently performed selective myotomy of the inner circular muscle from the esophagus to the gastric cardia ( $\bullet$ Fig. 2c). Neither remark- 
able bleeding nor a large vessel requiring further coagulation was encountered. We did however observe mucosal ischemic changes on the gastric cardia 1 hour after the submucosal tunnel had been created ( $\bullet$ Fig. 2d), by which time we had completed the succeeding myotomy and closed the mucosal entry with clips.

We administered intravenous broad spectrum antibiotics and a proton pump inhibitor to the fasting patient. After a further esophagography revealed no signs of perforation and his vital signs were stable, the patient resumed his diet without sequelae. A second-look endoscopy performed 1 month later revealed healing mucosa in the area of the previous lesion (๑ Fig. 3).

We suggest that injury to the vessel supplying blood to the gastric cardia during submucosal tunneling in addition to the effect of the epinephrine likely resulted in acute mucosal ischemia. Although this is a rare adverse event, an endoscopist should avoid extending dissection in the submucosal layer and unnecessarily coagulating visible vessels during POEM.

Endoscopy_UCTN_Code_CPL_1AH_2AK

Competing interests: None

\section{Chia-Wei Yang ${ }^{1}$, Bing-Yen Wang ${ }^{2}$, Kun-Ching Chou ${ }^{1}$, Hsu-Heng Yen ${ }^{1}$, Yang-Yuan Chen ${ }^{3}$}

${ }^{1}$ Department of Gastroenterology, Changhua Christian Hospital, Changhua, Taiwan

${ }^{2}$ Division of Thoracic Surgery, Department of Surgery, Changhua Christian Hospital, Changhua, Taiwan

${ }^{3}$ Department of Gastroenterology, China Medical University Hospital, Taichung, Taiwan

\section{Reference}

1 Inoue H, Minami H, Kobayashi Y et al. Peroral endoscopic myotomy (POEM) for esophageal achalasia. Endoscopy 2010; 42: $265-$ 271

Bibliography

DOI http://dx.doi.org/

10.1055/s-0035-1569655

Endoscopy 2015; 47: E614-E615

(C) Georg Thieme Verlag KG

Stuttgart · New York

ISSN 0013-726X

Corresponding author

Chia-Wei Yang, MD

Department of Gastroenterology

Changhua Christian Hospital

Changhua

Taiwan

Fax: +886-7-7228289

97601@cch.org.tw 